\title{
Oddballs and a Low Odderon Intercept
}

\author{
Felipe J. Llanes-Estrada ${ }^{1}$ * Pedro Bicudo ${ }^{2}$ 过 and Stephen R. Cotanch ${ }^{3}$ \\ ${ }^{1}$ Departamento de Física Teórica I, Universidad Complutense de Madrid, 28040 Madrid, Spain \\ ${ }^{2}$ Departamento de Física and CFIF, Instituto Superior Tecnico, Av. Rovisco Pais, 1049-001 Lisboa, Portugal \\ ${ }^{3}$ Department of Physics, North Carolina State University, Raleigh, NC 27695-8202
}

(Dated: October 9, 2018)

\begin{abstract}
We report an odderon Regge trajectory emerging from a field theoretical Coulomb gauge QCD model for the odd signature $J^{P C}(P=C=-1)$ glueball states (oddballs). The trajectory intercept is clearly smaller than the pomeron and even the $\omega$ trajectory's intercept which provides an explanation for the nonobservation of the odderon in high energy scattering data. To further support this result we compare to glueball lattice data and also perform calculations with an alternative model based upon an exact Hamiltonian diagonalization for three constituent gluons.
\end{abstract}

PACS numbers: 11.55.Jy, 12.39.Mk, 12.39.Pn, 12.40.Yx

Regge trajectories [1] have long been an effective phenomenological tool in hadronic physics. In Regge theory the scattering amplitude is governed by Regge poles, $\alpha_{n}(s)$, in the complex $J$ (angular momentum) plane. For integer $J$ the amplitude has a pole in the complex $s$ plane and, by crossing symmetry, for $t<0$ at high $s$ the cross section is dominated by the Regge trajectory, $\alpha(t)=b t+\alpha(0)$, with the largest intercept, $\alpha(0)$. This conjecture provides a unifying connection between hadron spectroscopy (Chew-Frautschi plot of $J$ vs. $t=M_{J}^{2}$ ) and the high energy behavior of the total cross section which scales as $s^{1-\alpha(0)}$. For elastic scattering the energy dependence is well described by the leading Regge trajectory, the pomeron, having $\alpha_{P}(0) \cong 1$ and $b_{P}=0.2-0.3 \mathrm{GeV}^{-2}$ (for recent fits see Ref. [2]). Of course the pomeron does not relate to conventional hadron spectra since meson trajectories typically have larger slopes, $b_{M} \cong .9 \mathrm{GeV}^{-2}$, and smaller intercepts, $\alpha_{M}(0) \cong .55$. According to the glueball-pomeron conjecture [3, 4], which is supported by lattice data [5] and other models [6], this trajectory is instead connected to glueball spectroscopy. Related, the different pomeron and meson trajectory slopes can be generated [4] by the gluon and quark color factors, respectively, used in confining potential models. Due to the large gluon mass gap, which suppresses relativistic corrections and transverse gluon exchange 7], these models tend to be more robust for glueballs than mesons. They produce a pomeron consisting of even signature $J^{++}$glueballs having maximum intrinsic spin $S$ coupled to minimum possible orbital $L$.

Of active interest is the odd signature, $P=C=-1$ counterpart to the pomeron, the odderon [8], for which there is no firm experimental evidence. Whereas the pomeron predicts asymptotically equal $p p$ and $\bar{p} p$ cross sections, the competitive presence of the odderon or any other $C=-1$ trajectory would produce a difference. However, high energy measurements reveal a minimal difference indicating that the odderon, if it exists, would have a smaller intercept probably at most comparable to the $\omega$ value, $\alpha_{\omega}(0)=0.5$. Indeed, dedicated exclusive searches at HERA [9] exclude an odderon Regge trajectory with an intercept greater than 0.7. Although perturbative QCD calculations 10 based on the BKP equation predict an odderon intercept close to 1 , they are only reliable for both $s,-t>>\Lambda_{Q C D}$ and thus suspect for $\alpha(t=0)$. For example, the predicted pomeron intercept using the similar BFKL equation is 1.5 in conflict with data.

In this paper we summarize recent nonperturbative QCD calculations based on lattice gauge theory and QCD models incorporating fundamental elements which produce realistic hadron spectra with Regge trajectories. Our key results clarify and dispel several misconceptions concerning the odderon. First, we document an odderon trajectory of odd signature $J^{--}$glueballs (oddballs) with intercept below 0.7 , consistent with experimental searches. Second, the odderon and pomeron have similar slopes, in analogy with the similar-slope meson (two-body) and baryon (three-body) Regge trajectories. Third, the lightest resonance on the odderon is the $3^{--}$, not the $1^{--}$which is on a daughter trajectory. In the framework of constituent models, the odderon begins with a $3^{--}$three gluon $L=0$ state with maximum spin $S$, similar to the two gluon pomeron beginning with the $\mathrm{s}$ wave, spin aligned $2^{++}$glueball. Higher odd signature states on the odderon have the same $S$ but increasing $L$ (e.g. d, g ... waves producing $J^{P C}=5^{--}, 7^{--} \ldots$ ).

The first constituent three gluon calculations [11, 12] utilized a non-relativistic potential model. They reported [12] the nearly degenerate lightest states, $J^{P C}=$ $0^{-+}, 1^{--}$and $3^{--}$, with masses about 4.8 times the constituent gluon mass. Hyperfine splittings breaking this degeneracy were considered in the bag model [13] but the global mass scale was too low [3]. Another problem confronting early constituent gluon models was a spurious two gluon $J=1$ state (unlike photons constituent gluons have 3 spin projections) which violates boson statistics (Yang's theorem). This issue is naturally resolved in the Coulomb gauge effective QCD formulation [14] in which the Fock operator commutation relations permit only transverse gluons respecting Yang's theorem. 
Our calculation is based on this relativistic many-body Hamiltonian formulation which unifies the quark and glue sectors. This approach dynamically generates constituent gluon and quark masses (while respecting chiral symmetry [15, 16]), produces reasonable quark and gluon condensates, describes flavored meson spectra, including hyperfine splittings 17, and predicts exotic hybrids 18] and $C=+1$ glueballs [4, 14] consistent with lattice gauge results. It is also noteworthy that this formulation entails only two dynamical parameters (same for both quark and glue sectors). Consult Refs. 16, 17, 19] for further details.

The effective QCD Hamiltonian in the gluon sector is

$$
\begin{aligned}
H_{e f f}^{g} & =\operatorname{Tr} \int d \mathbf{x}\left[\mathbf{\Pi}^{a}(\mathbf{x}) \cdot \mathbf{\Pi}^{a}(\mathbf{x})+\mathbf{B}_{A}^{a}(\mathbf{x}) \cdot \mathbf{B}_{A}^{a}(\mathbf{x})\right] \\
& -\frac{1}{2} \int d \mathbf{x} d \mathbf{y} \rho_{g}^{a}(\mathbf{x}) V(\mathbf{x}, \mathbf{y}) \rho_{g}^{a}(\mathbf{y}),
\end{aligned}
$$

with color charge density $\rho_{g}^{a}(\mathbf{x})=f^{a b c} \mathbf{A}^{b}(\mathbf{x}) \cdot \Pi^{c}(\mathbf{x})$, gauge fields $\mathbf{A}^{a}$, conjugate momenta $\boldsymbol{\Pi}^{a}=-\mathbf{E}^{a}$ and Abelian components $\mathbf{B}_{A}^{a}=\boldsymbol{\nabla} \times \mathbf{A}^{a}$, for $a=1,2, \ldots 8$. The normal mode expansions are

$$
\begin{aligned}
\mathbf{A}^{a}(\mathbf{x}) & =\int \frac{d \mathbf{q}}{(2 \pi)^{3}} \frac{1}{\sqrt{2 \omega_{k}}}\left[\mathbf{a}^{a}(\mathbf{q})+\mathbf{a}^{a \dagger}(-\mathbf{q})\right] e^{i \mathbf{q} \cdot \mathbf{x}}, \\
\boldsymbol{\Pi}^{a}(\mathbf{x}) & =-i \int \frac{d \mathbf{q}}{(2 \pi)^{3}} \sqrt{\frac{\omega_{k}}{2}}\left[\mathbf{a}^{a}(\mathbf{q})-\mathbf{a}^{a \dagger}(-\mathbf{q})\right] e^{i \mathbf{q} \cdot \mathbf{x}},
\end{aligned}
$$

with the Coulomb gauge transverse condition, $\mathbf{q} \cdot \mathbf{a}^{a}(\mathbf{q})=$ $(-1)^{\mu} q_{\mu} a_{-\mu}^{a}(\mathbf{q})=0$. Here $a_{\mu}^{a}(\mathbf{q}) \quad(\mu=0, \pm 1)$ are the bare gluon Fock operators from which, by a Bogoliubov-Valatin canonical transformation, the dressed gluon or quasiparticle operators, $\alpha_{\mu}^{a}(\mathbf{q})=$ $\cosh \Theta(q) a_{\mu}^{a}(\mathbf{q})+\sinh \Theta(q) a_{\mu}^{a \dagger}(-\mathbf{q})$, emerge. This similarity transformation is a hyperbolic rotation similar to the BCS fermion treatment. These operators excite constituent gluon quaisparticles from the BCS vacuum, $\Omega>>_{\mathrm{BCS}}$, and satisfy the transverse commutation relations, $\left[\alpha_{\mu}^{a}(\mathbf{q}), \alpha_{\nu}^{b \dagger}\left(\mathbf{q}^{\prime}\right)\right]=\delta_{a b}(2 \pi)^{3} \delta^{3}\left(\mathbf{q}-\mathbf{q}^{\prime}\right) D_{\mu \nu}(\mathbf{q})$, with $D_{\mu \nu}(\mathbf{q})=\left(\delta_{\mu \nu}-(-1)^{\mu} \frac{q_{\mu} q_{-\nu}}{q^{2}}\right)$. Finally, the quasiparticle or gluon self-energy, $\omega(q)=q e^{-2 \Theta(q)}$, satisfies a gap equation [14]. Both confinement and the leading QCD canonical interaction are contained in the Cornell type potential $V=\sigma r-\frac{\alpha_{s}}{r}$ with string tension, $\sigma=0.18 \mathrm{GeV}^{2}$, determined by lattice gauge calculations and $\alpha_{s}=0.42$. For this interaction the calculated gluon constituent mass from the gap equation, which uses the cut-off/renormalization parameter $\Lambda=1.1 \mathrm{GeV}$, is $m_{g} \equiv$ $\omega(0) \cong 0.8 \mathrm{GeV}$. Previous two constituent applications ( $q \bar{q}$ mesons and $g g$ glueballs) involved Tamm-Dancoff (TDA) and Random Phase approximation (RPA) Hamiltonian diagonalizations whereas three-body ( $q \bar{q} g$ hybrids) predictions utilized a variational calculation which was found to be accurate when tested in two-body systems. We therefore adopt the variational method for predicting the $g g g$ oddballs which is the minimum Fock space assignment for $C=-1$ glueballs. The three-gluon variational wavefunction is

$$
\begin{gathered}
\left|\Psi^{J P C}\right\rangle=\int d \mathbf{q}_{1} d \mathbf{q}_{2} d \mathbf{q}_{3} \delta\left(\mathbf{q}_{1}+\mathbf{q}_{2}+\mathbf{q}_{3}\right) \\
F_{\mu_{1} \mu_{2} \mu_{3}}^{J P C}\left(\mathbf{q}_{1}, \mathbf{q}_{2}, \mathbf{q}_{3}\right) C^{a b c} \alpha_{\mu_{1}}^{a \dagger}\left(\mathbf{q}_{1}\right) \alpha_{\mu_{2}}^{b \dagger}\left(\mathbf{q}_{2}\right) \alpha_{\mu_{3}}^{c \dagger}\left(\mathbf{q}_{3}\right)|\Omega\rangle_{\mathrm{BCS}}
\end{gathered}
$$

with summation over repeated indices. The color tensor $C^{a b c}$ is either totally antisymmetric $f^{a b c}$ (for $C=1$ ) or symmetric $d^{a b c}$ (for $C=-1$ ). Boson statistics thus requires the $C=-1$ oddballs to have a symmetric spacespin wavefunction taken here to have form

$$
\begin{gathered}
F_{\mu_{1} \mu_{2} \mu_{3}}^{J P C}\left(\mathbf{q}_{1}, \mathbf{q}_{2}, \mathbf{q}_{3}\right)=\left[c_{12} f\left(q_{1}, q_{2}\right)+c_{23} f\left(q_{2}, q_{3}\right)+\right. \\
\left.c_{13} f\left(q_{1}, q_{3}\right)\right]\left[Y_{L}^{\lambda}\left(\hat{\mathbf{q}}_{1}\right)+Y_{L}^{\lambda}\left(\hat{\mathbf{q}}_{2}\right)+Y_{L}^{\lambda}\left(\hat{\mathbf{q}}_{3}\right)\right], \\
c_{12}=\left\langle 1 \mu_{1} 1 \mu_{2} \mid s \mu_{s}\right\rangle\left\langle s \mu_{s} 1 \mu_{3} \mid S \mu\right\rangle\langle L \lambda S \mu \mid J \mathcal{M}\rangle,
\end{gathered}
$$

which is appropriate for this analysis of the lightest states which are either s or d wave oddballs with a single $L$ excitation. The other two coefficients in Eq. (5) can be obtained by permuting the indices in Eq. (6). A more comprehensive treatment would include other orbital angular excitations but since these are considerably separated in energy (more so than for conventional meson states) this mixing is small. Similar comments apply to higher radial wavefunction components. Several forms for the variational radial wavefunction, $f\left(q, q^{\prime}\right)$, involving two variational parameters, $\beta$ and $\beta^{\prime}$, were investigated including a separable form, $f(q) f\left(q^{\prime}\right)$, which only involves one parameter. From previous experience [18], reliable, accurate variational solutions can be obtained if these functions have a bell-shaped form with scalable variational parameters. In particular we found numerical solutions of the $\rho$ meson TDA problem for the same potential to be especially accurate.

The variational equation for the $J^{P C}$ glueball with mass $M_{J P C}$ is

$$
\frac{\left\langle\Psi^{J P C}\left|H_{e f f}^{g}\right| \Psi^{J P C}\right\rangle}{\left\langle\Psi^{J P C} \mid \Psi^{J P C}\right\rangle}=M_{J P C} .
$$

For clarity and insight it is worthwhile to express $H_{\text {eff }}^{g}=$ $H_{s e}+H_{s c}+H_{a n}$ as a sum of three terms corresponding to self-energy (two-body contributions), $H_{s e}$, scattering (instantaneous exchange interaction between two gluons), $H_{s c}$, and annihilation, $H_{a n}$, of two gluons. Figure 1 depicts the three-gluon diagram for each interaction. The contribution from the self-energy term is

$$
\begin{gathered}
\frac{\left\langle\Psi\left|H_{s e}\right| \Psi\right\rangle}{\langle\Psi \mid \Psi\rangle}=18 \int d \mathbf{q}_{1} d \mathbf{q}_{2} F_{\mu_{1} \mu_{2} \mu_{3}}^{*}\left(\mathbf{q}_{1}, \mathbf{q}_{2}\right) F_{\nu_{1} \nu_{2} \nu_{3}}\left(\mathbf{q}_{1}, \mathbf{q}_{2}\right) \\
D_{\mu_{1} \alpha}\left(\mathbf{q}_{1}\right) D_{\mu_{2} \nu_{2}}\left(\mathbf{q}_{2}\right) D_{\mu_{3} \nu_{3}}\left(\mathbf{q}_{3}\right) D_{\beta \nu_{1}}\left(\mathbf{q}_{1}\right)\left[\frac{\omega_{1}^{2}+q_{1}^{2}}{2 \omega_{1}} \delta_{\alpha \beta}\right. \\
\left.\quad-\frac{3}{4} \int \frac{d \mathbf{q}}{(2 \pi)^{3}} \hat{V}(q) \frac{\omega_{1}^{2}+\omega_{6}^{2}}{\omega_{1} \omega_{6}} D_{\alpha \beta}\left(\mathbf{q}_{6}\right)\right],
\end{gathered}
$$

where we have suppressed the $J^{P C}$ superscript and the dependent variable $\mathbf{q}_{3}=-\mathbf{q}_{1}-\mathbf{q}_{2}$. Again $\omega_{i}=\omega\left(\mathbf{q}_{i}\right)$ is 

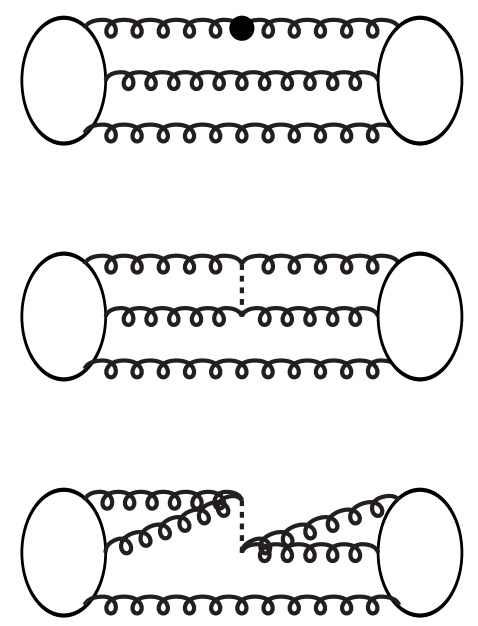

FIG. 1: Interaction diagrams for the $g g g$ state in the $H_{\text {eff }}^{g}$ approach. Top to bottom corresponds to the gluonic selfenergy, scattering and annihilation interactions, respectively.

the solution to the gluon gap equation [4, 16], $\mathbf{q}_{6}=\mathbf{q}_{1}+\mathbf{q}$ and $\mathbf{q}$ is the momentum transferred by the interaction. Similarly the scattering contribution is

$$
\begin{gathered}
\frac{\left\langle\Psi\left|H_{s c}\right| \Psi\right\rangle}{\langle\Psi \mid \Psi\rangle}=-\frac{9}{2} \mathcal{F}_{s c}^{ \pm} \int d \mathbf{q}_{1} d \mathbf{q}_{2} d \mathbf{q} F_{\mu_{1} \mu_{2} \mu_{3}}^{*}\left(\mathbf{q}_{1}, \mathbf{q}_{2}\right) \hat{V}(q) \\
F_{\nu_{1} \nu_{2} \nu_{3}}\left(\mathbf{q}_{4}, \mathbf{q}_{5}\right) \frac{\left(\omega_{1}+\omega_{4}\right)\left(\omega_{2}+\omega_{5}\right)}{\sqrt{\omega_{1} \omega_{2} \omega_{4} \omega_{5}}} D_{\mu_{3} \nu_{3}}\left(\mathbf{q}_{3}\right) D_{\mu_{2} \beta}\left(\mathbf{q}_{2}\right) \\
D_{\beta \nu_{2}}\left(\mathbf{q}_{5}\right) D_{\mu_{1} \alpha}\left(\mathbf{q}_{1}\right) D_{\alpha \nu_{1}}\left(\mathbf{q}_{4}\right)
\end{gathered}
$$

with $\mathbf{q}_{4}=\mathbf{q}_{1}-\mathbf{q}, \mathbf{q}_{5}=\mathbf{q}_{2}+\mathbf{q}$. Finally the annihilation contribution is

$$
\begin{gathered}
\frac{\left\langle\Psi\left|H_{a n}\right| \Psi\right\rangle}{\langle\Psi \mid \Psi\rangle}=\frac{9}{4} \mathcal{F}_{a n}^{ \pm} \int d \mathbf{q}_{1} d \mathbf{q}_{2} d \mathbf{q} F_{\mu_{1} \mu_{2} \mu_{3}}^{*}\left(\mathbf{q}_{1}, \mathbf{q}_{7}\right) \hat{V}(q) \\
F_{\nu_{1} \nu_{2} \nu_{3}}\left(\mathbf{q}_{8}, \mathbf{q}_{2}\right) \frac{\left(\omega_{1}+\omega_{7}\right)\left(\omega_{2}+\omega_{8}\right)}{\sqrt{\omega_{1} \omega_{2} \omega_{7} \omega_{8}}} D_{\mu_{3} \nu_{3}}(\mathbf{q}) D_{\mu_{1} \alpha}\left(\mathbf{q}_{1}\right) \\
D_{\mu_{2} \alpha}\left(\mathbf{q}_{7}\right) D_{\nu_{1} \beta}\left(\mathbf{q}_{8}\right) D_{\beta \nu_{2}}\left(\mathbf{q}_{2}\right), \quad(10)
\end{gathered}
$$

with $\mathbf{q}_{7}=\mathbf{q}-\mathbf{q}_{1}$ and $\mathbf{q}_{8}=\mathbf{q}-\mathbf{q}_{2}$. The first two contributions exactly cancel the infrared singularity in the instantaneous potential. The color factors $\mathcal{F}^{C= \pm}$ depend upon the $C$ parity and follow directly from the expectation value of the density-density $\left(f^{a b c} f^{a e f}\right)$ term in the Coulomb kernel: $\mathcal{F}_{s c}^{+}=\frac{f^{a b c} f^{a e f} f^{b e i} f^{c f i}}{f^{a b c} f^{a b c}}=$ $\frac{-36}{24}=-\frac{3}{2}, \mathcal{F}_{s c}^{-}=\frac{d^{a b c} d^{a e f} f^{b e i} f^{c f i}}{d^{a b c} d^{a b c}}=\frac{-20}{40 / 3}=-\frac{3}{2}, \mathcal{F}_{a n}^{+}=$ $\frac{f^{a b c} f^{a e f} f^{b c i} f^{e f i}}{f^{a b c} f^{a b c}}=\frac{72}{24}=3$ and $\mathcal{F}_{a n}^{-}=\frac{d^{a b c} d^{a e f} f^{b c i} f^{e f i}}{d^{a b c} d^{a b c}}=$ $\frac{0}{40 / 3}=0$, using the orthogonality relation $d^{a b c} f^{a b c}=0$ and computer algebra (FORM 20]). The $H_{a n}$ interaction splits the $0^{-+} \mathrm{s}$ wave glueball state from the $1^{--}$and $3^{--}$ levels, all calculated degenerate in the work of Ref. [12]. Also, annihilation is not present in simple $g g g$ potential models as well as all models for $g g$ states which is why it has not been studied previously in investigations of the lightest $0^{-+}$mass, involving $\mathrm{p}$ wave two-gluon states, calculated between $2.1 \mathrm{GeV}[14$ and $2.6 \mathrm{GeV}[21]$.

The variational calculation entails nine-dimensional integrals which where performed using the Monte Carlo method with the adaptive sampling algorithm VEGAS 22]. In general, numerical convergence was achieved with about $10^{5}$ samples. A study of the glueball mass sensitivity to both statistical and variational uncertainties yielded error bars at the 3 to $5 \%$ level.

For comparison we also calculated oddball masses using a simpler nonrelativistic constituent model Hamiltonian 23.

$$
H_{M}=\sum_{i} \frac{\mathbf{q}_{i}^{2}}{2 m_{g}}+V_{0}+\sum_{i<j}\left[\sigma r_{i j}-\frac{\alpha}{r_{i j}}+V_{s s} \mathbf{S}_{i} \cdot \mathbf{S}_{j}\right],(11)
$$

with $r_{i j}=\left|\mathbf{r}_{i}-\mathbf{r}_{j}\right|$ and parameters taken from the $q \bar{q}$ funnel potential: $V_{0}=-0.90 \mathrm{GeV}, \alpha=0.27, \sigma=0.25 \mathrm{GeV}^{2}$. The gap equation gluon mass value, $m_{g}=0.8 \mathrm{GeV}$, is also used. Exactly diagonalizing, $H_{M} \Psi^{J P C}=M_{J P C} \Psi^{J P C}$, and only adjusting $V_{s s}=0.085 \mathrm{GeV}$ to optimize agreement with lattice, yields the predicted $J^{++}$glueball Regge trajectory, $\alpha_{P}^{M}=0.23 t+1.0$, consistent with the pomeron. In the $g g$ glueball calculation an algebraic color factor of 2 multiplies each bracketed term in the potential. With these fixed parameters the $g g g$ oddball spectrum was then obtained by exact diagonalization using Jacobi coordinates and an expansion in a harmonic oscillator basis. Further details will be reported in a future communication. The glueball masses and quantum numbers investigated are listed in Table \ Note that the $H_{\text {eff }}^{g}$ ground state for the $g g g$ glueball is technically not an oddball but the $0^{-+}$with mass $3900 \mathrm{MeV}$. Also, the model $g g g$ glueballs are heavier than $g g$ states (both having low excitations) which is consistent with predictions deduced from the holographic dual theory of QCD 24].

TABLE I: Glueball quantum numbers and masses in $\mathrm{MeV}$.

\begin{tabular}{|c|c|cccccc|}
\hline \hline Model & $J^{P C}$ & $0^{-+}$ & $1^{--}$ & $2^{--}$ & $3^{--}$ & $5^{--}$ & $7^{--}$ \\
\hline this work & color & $f$ & $d$ & $d$ & $d$ & $d$ & $d$ \\
& $S$ & 0 & 1 & 2 & 3 & 3 & 3 \\
& $L$ & 0 & 0 & 0 & 0 & 2 & 4 \\
$H_{\text {eff }}^{g}$ & & 3900 & 3950 & 4150 & 4150 & 5050 & 5900 \\
$H_{M}$ & & 3400 & 3490 & 3660 & 3920 & 5150 & 6140 \\
\hline lattice [21] & & 3640 & 3850 & 3930 & 4130 & \\
lattice [5] & & 3250 & 3100 & 3550 & 4150 & & \\
Wilson loops [25] & & 3770 & 3490 & 3710 & 4030 & & \\
\hline \hline
\end{tabular}

Figure 2 summarizes our key findings and displays predicted oddball Regge trajectories from the alternative approaches. Lattice results are represented by diamonds [5] and open circles [21], while boxes [25] are constituent model predictions using a Wilson-loop inspired potential. Solid circles and triangles correspond 


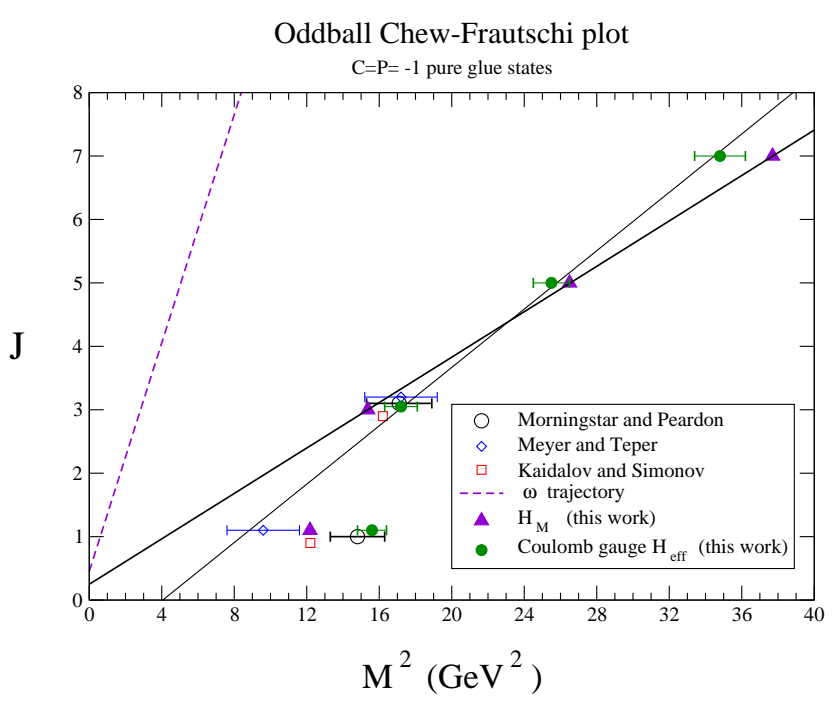

FIG. 2: Odderon trajectory from three-gluon spectroscopy and lattice compared to the $\omega$ meson Regge trajectory.

to the $H_{\text {eff }}^{g}$ and $H_{M}$ models, respectively. The resulting odderon trajectories are represented by the solid lines, $\alpha_{O}^{e f f}=0.23 t-0.88$ and $\alpha_{O}^{M}=0.18 t+0.25$, whose differences provide an overall theoretical uncertainty. The dashed line is the $\omega$ trajectory.

Several conclusions follow. First, this work predicts an odderon having slope similar to the pomeron but intercept even lower than the $\omega$ value. Second, the first odderon state is the $3^{--}$and not the $1^{--}$which falls on a daughter trajectory. Unfortunately the other approaches did not report a $5^{--}$glueball which could confirm this point. Since at least two points are necessary to establish a trajectory we strongly recommend that future studies calculate higher $J$ states. Lastly, there appears to be general model consensus that the $3^{--}$mass is close to $4 \mathrm{GeV}$ and also support for the $0^{-+}$as a ground state candidate for the $g g g$ system.

In summary, we have compared existing oddball predictions to our large-scale model evaluations for $J^{--}$ glueballs. The results document an odderon trajectory subdominant to the pomeron which can explain its nonobservance in reactions with pomeron exchange. Should the odderon intercept be comparable to the $\omega$ value it may be possible to see it in pseudoscalar [26] or tensor meson 27] electromagnetic production where the pomeron is absent. However if the intercept is below 0.5, as we predict, it is unlikely the odderon will be observed.

F.L. acknowledges a Fundacion del Amo-Univ. Complutense fellowship and the hospitality of the SLAC the- ory group. Thanks to E. Abreu, S. Brodsky, J. Vary and P. Zerwas for useful conversations. Research supported by Spanish Grant MCYT FPA 2004-02602 (F.L.) and U. S. DOE Grant DE-FG02-97ER41048 (S.C.).

* On leave at: Theory Group, Stanford Linear Accelerator Center, 2571 Sand Hill Rd. 94025 Menlo Park CA; Electronic address: fllanes@fis.ucm.es

$\dagger$ Electronic address: bicudo@ist.utl.pt

\# Electronic address: cotanch@ncsu.edu

[1] T. Regge, Nuovo Cim. 14, 951 (1959).

[2] J. R. Pelaez and F. J. Yndurain, Phys. Rev. D 69, 114001 (2004).

[3] Y. A. Simonov, Phys. Lett. B 249, 514 (1990).

[4] F. J. Llanes-Estrada, S. R. Cotanch, P. Bicudo, J. F. T. Ribeiro and A. Szczepaniak, Nucl. Phys. A 710, 45 (2002) arXiv:hep-ph/0008212.

[5] H. B. Meyer and M. Teper, Phys. Lett. B 605, 344 (2005).

[6] F. Brau and C. Semay, Phys. Rev. D 70, 014017 (2004).

[7] R. Alkofer, C. S. Fischer and F. J. Llanes-Estrada, Phys. Lett. B 611, 279 (2005).

[8] L. Lukaszuk and B. Nicolescu, Nuovo Cim. Lett. 8, 405 (1973).

[9] C. Adloff, et al. (H1 Collaboration), Phys. Lett. B 544, 35 (2002).

[10] C. Ewerz, arXiv:hep-ph/0306137

[11] J. Cornwall and A. Soni, Phys. Lett. B 120, 431 (1983).

[12] W. S. Hou and A. Soni, Phys. Rev. D 29, 101 (1984).

[13] T. Barnes, F. E. Close and S. Monaghan, Nucl. Phys. B 198, 380 (1982).

[14] A. Szczepaniak, E. S. Swanson, C. R. Ji and S. R. Cotanch, Phys. Rev. Lett. 76, 2011 (1996).

[15] F. J. Llanes-Estrada and S. R. Cotanch, Phys. Rev. Lett. 84, 1102 (2000).

[16] F. J. Llanes-Estrada and S. R. Cotanch, Nucl. Phys. A. 697, 303 (2002).

[17] F. J. Llanes-Estrada, S. R. Cotanch, A. Szczepaniak and E. S. Swanson, Phys. Rev. C 70, 035202 (2004).

[18] F. J. Llanes-Estrada and S. R. Cotanch, Phys. Lett. B 504, 15 (2001).

[19] S. R. Cotanch, Prog. Part. Phys. 50, 353 (2003).

[20] J. A. M. Vermaseren, arXiv:math-ph/0010025

[21] C. J. Morningstar and M. Peardon, Phys. Rev. D 60, 034509 (1999).

[22] G. P. Lepage, Cornell preprint CLNS 80-447 (1980).

[23] P. Bicudo, The Second PANDA Physics Workshop, Laboratori Nazionali di Frascati, March 18-19, Frascati, (2004) arXiv:hep-ph/0405223.

[24] G. F. de Teramond and S. J. Brodsky, Phys. Rev. Lett. 94, 201601 (2005).

[25] A. B. Kaidalov and Y. A. Simonov, Phys. Lett. B 477, $163(2000)$.

[26] E. R. Berger, et al., Eur. Phys. J. C 9, 491 (1999).

[27] E. R. Berger, et al., Eur. Phys. J. C 14, 673 (2000). 